\title{
Sudan Black B masks Mycobacterium avium subspecies paratuberculosis immunofluorescent antibody labeling
}

Caitlin J. Jenvey and Judith R. Stabel ${ }^{*}$

*Correspondence: judy.stabel@ars.usda.gov

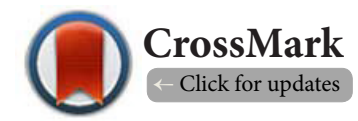

USDA-Agricultural Research Service (ARS), National Animal Disease Center, Ames, lowa, United States of America.

\begin{abstract}
Intestinal lipofuscin is particularly problematic for immunofluorescence protocols as it is inherently fluorescent. Sudan Black B (SBB) has been used successfully in the past to mask lipofuscin autofluorescence, as well as for staining of mycobacteria. Mycobacteria produce unique cell-wall lipids; therefore, SBB may not be suitable for inclusion in an immunofluorescence protocol that includes a Mycobacterium avium subspecies paratuberculosis (MAP) primary antibody. The effect of SBB, when compared to 3,3'-diaminobenzidine (DAB), on immunofluorescent antibody labeling was compared for MAP-specific antibody labeled, and TLR4specific antibody labeled frozen bovine intestinal tissue sections. The effect of SBB on immunofluorescent antibody labeling when added prior to the primary antibody or after the secondary antibody was also compared. The masking ability of SBB was also compared with DAB for unlabeled tissue sections. When compared to DAB only treated tissue, SBB added before the primary or after the secondary antibody reduced the intensity and abundance of immunofluorescently labeled MAP. We recommend that the use of SBB be excluded from immunofluorescence protocols that utilize a MAP-specific primary antibody in order to optimize immunofluorescent labeling.
\end{abstract}

Keywords: Bovine, eosinophil, frozen, intestine, lipofuscin, Mycobacterium avium subsp, paratuberculosis, Sudan Black

\section{Introduction}

The intestine is home to a number of inherently fluorescent complexes. Lipofuscin is particularly problematic as it accumulates in the cytoplasm of cells located in the lamina propria, alongside cells and structures of interest, and is visible under all common fluorescence filters [1]. A simple method of reducing this autofluorescence is to quench it with Sudan Black B (SBB). Sudan Black B is a black, histochemical stain that binds to lipids in tissue and masks lipofuscin autofluorescence, rather than altering it on a chemical level [1]. A number of studies have used SBB to successfully quench lipofuscin autofluorescence in tissue [1-4], however it is unclear whether its inclusion in an immunofluorescence protocol may also mask immunofluorescent antibody labeling for specific pathogens such as Mycobacterium avium subspecies paratuberculosis (MAP). Mycobacteria are known to produce ubiquitous phospholipids and distinct genus-specific lipids [5]. On average, the lipid content of mycobacteria is approximately $15 \%$, but can be as high as $60 \%$ within the cell wall [6]. Previous studies have incorporated
SBB staining of Mycobacterium avium subspecies avium(MAA) [7,8], M. leprae [7,8], M. bovis, M. tuberculosis, Mycobacterium (unspecified cold-blooded species), M. phlei, M. smegmatis, and Mycobacterium sp. (saprophytic) [8]. Studies by Ortalo-Magne [9] and Naser [10] have demonstrated glycolipoproteins on the outer-most region of the cell envelope in MAA and MAP, respectively, that was confirmed by SBB staining in the study by Naser [10].

The aim of the present study was to determine whether the inclusion of SBB in an immunofluorescence protocol would mask MAP-specific immunofluorescent antibody labeling in frozen bovine intestinal tissue sections collected from dairy cattle naturally infected with MAP. We concluded that SBB resulted in an unacceptable reduction in MAP-specific immunofluorescent labeling and quality.

\section{Materials and methods \\ Snap-freezing protocol}

A dry ice bath was prepared by combining $95 \%$ ethanol with 
dry ice and mixed until a slurry consistency was achieved. Isopentane (Sigma-Aldrich, St. Louis, Missouri) was added to a tin cup and the cup was placed into the dry ice bath. A midileal intestinal sample from 1 cow naturally infected with MAP was obtained at necropsy. The cow tissue used in the current study was selected based upon the presence of a high level of tissue autofluorescence when compared to other cow tissues within a cohort used for experimental study. The ileal section was washed with PBS, pH 7.4, cut open and positioned lumen side down on top of a liver sample covered with Tissue-Tek O.C.T. (Sakura Finetek, Torrance, California).The intestine-liver sample was wrapped in foil and placed in the isopentane for at least 5 minutes. The snap-frozen sample was transferred to dry ice for transport to storage at $-80 \mathrm{C}$, where it remained until tissue sectioning could be performed.

\section{Tissue sections}

The mid-ileal intestinal sample was removed from $-80 \mathrm{C}$ and placed in a cryostat at $-20 \mathrm{C}$ for at least 30 minutes prior to sectioning. The tissue sample was embedded in Tissue-Tek O.C.T. and cut in $6 \mu \mathrm{m}$ sections and adhered to Color Frost Plus microscope slides (Thermo Fisher Scientific, Carlsbad, California). Tissue sections were allowed to air-dry overnight at room temperature before fixing for 5 minutes at $-20 \mathrm{C}$. Tissue sections were stored at $-80 \mathrm{C}$ until immunofluorescence staining could be performed.

\section{Immunofluorescence protocol}

The effect of SBB on immunofluorescent labeling was compared using two primary antibodies: a heat-killed MAP rabbit polyclonal diluted 1:1000 (in-house), and a TLR4 rabbit polyclonal diluted 1:200 (Bioss Antibodies, Woburn, Massachusetts). The primary antibodies were fluorescently labeled with a cross-adsorbed Alexa Fluor 647 goat anti-rabbit IgG secondary antibody (ThermoFisher Scientific, Carlsbad, California), diluted 1:1000.

Tissue sections were removed from $-80 \mathrm{C}$ and allowed to equilibrate to room temperature for 10-20 minutes. A liquid blocker 'Pap' pen was used to draw a hydrophobic barrier around the tissue and allowed to dry. Tissue sections were rehydrated with $400 \mu \mathrm{L}$ of $0.05 \mathrm{M}$ Tris buffer for 10 minutes, followed by quenching of autofluorescence with $100 \mu \mathrm{L}$ of 3,3'-diaminobenzidine (DAB) (Vector Laboratories, Burlingame, California) for 10 minutes. Slides were washed for 5 minutes in $0.05 \mathrm{M}$ Tris buffer with $0.2 \%$ Tween- 20 and $0.9 \% \mathrm{NaCl}$. Tissue sections were blocked with $100 \mu \mathrm{L}$ of $10 \%$ normal equine serum in Tris buffer for 30 minutes. The slides were not rinsed in-between the blocking and primary antibody incubation steps.The primary and secondary antibodies were incubated for 60 minutes at room temperature in a humidified chamber. Following antibody incubation, the slides were washed three times for 5 minutes, alternating between $0.05 \mathrm{M}$ Tris buffer and $0.05 \mathrm{M}$ Tris buffer with $0.2 \%$ Tween -20 and $0.9 \% \mathrm{NaCl}$. Slides were mounted in ProLong Gold Antifade Mountant
(ThermoFisher Scientific, Carlsbad, California) and RichardAllen Scientific 'Slip-Rite' Cover Glass \#1.5 (Thermo Fisher Scientific, Carlsbad, California). The mounting medium was allowed to cure for at least 30 minutes at room temperature before imaging.

\section{Sudan Black B protocol}

Sudan Black B (SBB) (Eastman Kodak Company, Rochester, New York) was prepared as a $0.3 \%$ solution in $70 \%$ ethanol stirred in the dark for 2 hours (3). Tissue sections were incubated with $100 \mu \mathrm{L} \mathrm{SBB}$ for 10 minutes at room temperature. The slides were rinsed with 70\% ethanol and washed for 5 minutes in $0.05 \mathrm{M}$ Tris buffer with $0.2 \%$ Tween- 20 and $0.9 \% \mathrm{NaCl}$. The SBB treated slides were compared to $D A B$ for its ability to also mask inherent tissue autofluorescence. The DAB was prepared as per the manufacturer's instructions in $5 \mathrm{~mL}$ of deionized water. To assess whether the point at which SBB was included in an immunofluorescence protocol could affect labeling, tissue was treated with SBB before blocking and primary antibody incubation (Before), and compared to tissue treated with SBB after the secondary antibody (After). Quenching protocols were compared for unlabeled and labeled tissue as follows: unlabeled+untreated, unlabeled $+\mathrm{DAB}$, unlabeled $+\mathrm{SBB}$, unlabeled+DAB+SBB, MAP+DAB, MAP+DAB+SBB (Before), $M A P+D A B+S B B$ (After), TLR4+DAB, TLR4+DAB+SBB (Before), and TLR4+DAB+SBB (After).

\section{Confocal Imaging}

Imaging was performed using a Nikon A1 Resonance plus inverted microscope equipped with a four-laser GalliumArsenide-Phosphide/normal Photomultiplier Tube detector unit (DU4) (GaAsP: 488 and 561; PMT: 405 and 640), Galvano resonant scanner and NIS Elements Advanced Research software (version 4.50.00). Images were acquired by sequential scanning to avoid fluorescence cross-over using a 405/488/561/640 dichroic mirror. All slides were imaged using a $488 \mathrm{~nm}$ solid-state diode laser and $525 / 50$ bandpass filter, and 561 solid-state diode laser and 600/50 bandpass filter, and a 640 solid-state diode laser and $685 / 70$ bandpass filter for labeled tissue. All images were captured using a 20x Plan Apo lambda objective (1024 x 1024 pixels), pinhole $1.2 \mathrm{AU}$, numerical aperture 0.75 , and exposure 6.2 seconds per pixel dwell. Detector sensitivity (gain) and laser power settings, for each laser, were kept the same for all collected images to allow comparisons between images and treatments. Upon collection of each image, the NIS Elements Advanced Research software automatically calculates the mean fluorescence intensity for the whole image (measured in number of pixels). A total of 5 images per slide were collected to perform statistical analysis.

\section{Statistical analysis}

Descriptive statistics, including mean and $95 \%$ confidence intervals (Cl) $(95 \% \mathrm{Cl}$ were calculated using standard deviation and standard error) were calculated for each treatment, 
Jenvey et al. Journal of Histology \& Histopathology 2017,

per antibody, using Microsoft Excel for Mac (2011).

\section{Results}

The mean fluorescence intensity for the unlabeled+untreated tissue was significantly higher when compared to all treated slides (Table 1). The mean fluorescence intensity for the unlabeled+SBB tissue was significantly lower $(89.8 \pm 0.7395 \%$ C.I.) when compared to unlabeled+DAB $(156.0 \pm 3.3495 \%$ C.I.) and unlabeled+DAB+SBB (113.8 $\pm 1.4495 \%$ C.I.) treated tissue (Table 1).

For tissue labeled with the MAP-specific primary antibody, $D A B$ treated tissue demonstrated the highest mean fluorescence intensity when compared to $D A B+S B B$ (Before) and $\mathrm{DAB}+\mathrm{SBB}$ (After) (Table 2). For tissue treated with $\mathrm{DAB}+\mathrm{SBB}$ (Before) and $\mathrm{DAB}+\mathrm{SBB}$ (After), mean fluorescence intensity was reduced by $15 \%$ and $7 \%$, respectively, when compared to tissue treated with $D A B$ only. For tissue labeled with a TLR4-specific primary antibody, DAB treated tissue also demonstrated the highest mean fluorescence intensity when compared to DAB+SBB (Before) and DAB+SBB (After) (Table 2). For tissue treated with $D A B+S B B$ (Before) and $D A B+S B B$ (After), mean fluorescence intensity was reduced by $17 \%$ and $25 \%$, respectively, when compared to tissue treated with $D A B$ only. The observed differences between treatments were not significant $(P>0.05)$.

Immunofluorescent antibody labeling was observed for both primary antibodies (Figure 1). There was no obvious difference in the abundance of TLR4-specific immunofluorescent antibody labeling between the three treatments (Figures 1A-1C).
Tissue treated with $\mathrm{DAB}+\mathrm{SBB}$ (Before) (Figure $1 \mathrm{E}$ ) and $\mathrm{DAB}+\mathrm{SBB}$ (After) (Figure 1F) demonstrated an observable reduction in the abundance of MAP-specific immunofluorescent antibody labeling, when compared to tissue treated with DAB only (Figure 1D).

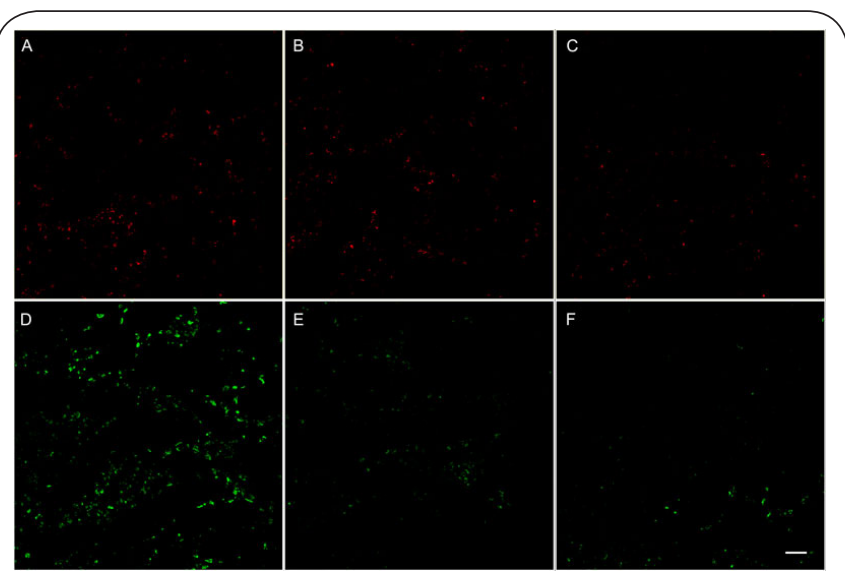

Figure 1. Immunofluorescent antibody labeling of MAP and TLR4 in frozen bovine mid-ileal intestinal tissue collected from a cow naturally infected with MAP. Tissue sections were treated as follows: TLR4 DAB only $(\mathbf{A})$, TLR4 DAB+SBB(Before) (B), TLR4 DAB+SBB (After)(C), MAP DAB only (D), MAP DAB+SBB (Before) (E), and MAP DAB+SBB (After)(F). Scale bar $=50$ microns. MAP: Mycobacterium avium subspecies paratuberculosis; TLR4: Toll-like Receptor 4; DAB: 3,3'-diaminobenzidine; SBB: Sudan Black B.

Table 1. Mean ( $\pm 95 \%$ C.I.) fluorescence intensity (pixels) for unlabeled frozen mid-ileal intestinal tissue collected from a cow naturally infected with MAP, for untreated DAB only, SBB only and DAB + SBB only treated tissue, $(n=5)$.

\begin{tabular}{llllll}
\hline \multicolumn{5}{c}{ Unlabeled Tissue } \\
\hline Excitation & Emission & Untreated & DAB Only & SBB Only & DAB+SBB \\
\hline 488 & $475-575$ & $207.2 \pm 9.31$ & $156.0 \pm 3.34$ & $89.8 \pm 0.73$ & $113.8 \pm 1.44$ \\
561 & $550-650$ & $181.6 \pm 8.80$ & $97.6 \pm 1.00$ & $75.8 \pm 0.73$ & $105.4 \pm 2.37$ \\
\hline
\end{tabular}

MAP: Mycobacterium avium subspecies paratuberculosis;

DAB: 3,3'-diaminobenzidine; SBB: Sudan Black B

Table 2. Mean ( $\pm 95 \%$ C.I.) fluorescence intensity (pixels) for MAP and TLR4 immunofluorescently labeled with Alexa Fluor 647 in frozen mid-ileal intestinal tissue collected from a cow naturally infected with MAP, for DAB only, DAB+SBB (Before) and $\mathrm{DAB}+\mathrm{SBB}$ (After) treated tissue, $(\mathrm{n}=5)$.

\begin{tabular}{lll}
\hline & \multicolumn{2}{l}{ Primary Antibody } \\
\hline Quenching Protocol & MAP & TLR4 \\
\hline DAB Only & $166.4 \pm 17.73$ & $181.6 \pm 11.67$ \\
DAB+SBB (Before) & $142.0 \pm 6.50$ & $150.2 \pm 26.67$ \\
DAB+SBB (After) & $154.4 \pm 23.62$ & $137.0 \pm 17.23$ \\
\hline
\end{tabular}

MAP: Mycobacterium avium subspecies paratuberculosis;

DAB: 3,3'-diaminobenzidine; SBB: Sudan Black B 
Jenvey et al. Journal of Histology \& Histopathology 2017,

http://www.hoajonline.com/journals/pdf/2055-091X-4-11.pdf

doi: 10.7243/2055-091X-4-11

\section{Discussion}

The inclusion of SBB in an immunofluorescence protocol did mask MAP-specific immunofluorescent antibody labeling, however, the point at which it was included in the protocol determined the degree to which immunofluorescent antibody labeling was reduced. When included in the protocol prior to incubation with the primary antibody, SBB quenched the intensity of immunofluorescent signal associated with antibody-labeled MAP by $15 \%$, which was greater when compared to the reduction in signal obtained when SBB was added after the secondary antibody (7\% reduction).The difference in the ability of SBB to reduce the abundance of fluorescently labeled MAP when added before the primary, and after the secondary antibody, is likely due to the MAPspecific antibody used in this study. The antibody was a polyclonal antibody raised in rabbit against heat-killed MAP, created from a MAP pellet containing $5 \mathrm{mg}$ of cell wall protein per milliliter [11]. It is likely that the addition of SBB before the primary antibody incubation allowed the SBB to bind to lipids in the MAP cell wall present within the infected tissues, limiting the ability of the MAP-specific primary antibody to bind to cell wall proteins. In contrast, adding SBB upon completion of primary and secondary antibody labeling allowed the MAP-specific primary antibody to bind to available MAP cell wall protein sites. This would allow SBB to bind to any remaining lipid binding sites, reducing, but not eliminating, MAP immunofluorescent labeling. In contrast, the intensity of immunofluorescent signal associated with antibody-labeled TLR4 was quenched by $25 \%$ when adding SBB after the secondary antibody, with less quenching observed in signal obtained when SBB was added before the primary antibody (17\% reduction). Despite these reductions in fluorescence intensity, there was no apparent difference in the observed immunofluorescent labeling of TLR4 (Figures 1A-1C). Although the effectiveness of SBB to mask lipofuscin autofluorescence whilst maintaining immunofluorescent labeling is concentration dependent, it is unlikely that the concentration used in this study was the basis for this difference in intensity. In a study by Schnell [1], SBB concentrations between 1-10\% eliminated all autofluorescent pigments, however, this came at the expense of specific immunofluorescent labeling. This study found that SBB concentrations less than 1\% allowed the visualization of the fluorophores tested, including Cy5 [1], which demonstrates similar excitation and emission wavelengths to the Alexa Fluor 647 used in the current study.

It should also be noted that although SBB reduced inherent tissue autofluorescence further when compared to tissue treated with DAB only, it is likely that this difference between treatments is not due to their masking ability, but rather, due to the source of the autofluorescence in the tissue sections. While SBB masks lipids, DAB masks eosinophils by reacting with endogenous peroxidase [12], which is contained within the cytoplasmic granules of the eosinophil. The authors noticed that autofluorescence thought to be due to lipofuscin was more abundant and more fluorescent than autofluorescence thought to be due to eosinophils (data not shown). It is most likely that the tissue sections used in this study were more abundant in lipofuscin than eosinophils, thus SBB, or a combination of SBB and DAB, demonstrated a better reduction of tissue autofluorescence when compared to DAB only. Additionally, the efficiency of SBB to mask autofluorescence may have been impacted by cow-to-cow variability in the abundance of lipofuscin or eosinophils, as well as within-tissue variation in the location of the autofluorescent compounds. However, contiguous frozen sections were taken to best of our ability for the purposes of comparisons between the two primary targets, MAP and TLR4.

In summary, we demonstrated that using SBB to quench autofluorescence also results in the masking of immunofluorescent MAP-specific antibody labeling in frozen bovine intestinal tissue sections, however, these results were not statistically significant. Further studies using a larger sample size and/or more images may show such a difference. Despite this lack of statistical difference between treatments, we recommend that SBB be excluded from immunofluorescence protocols that utilize a MAP-specific primary antibody in order to optimize immunofluorescent labeling. However, for protocols that do not use a MAP-specific primary antibody, and autofluorescence is deemed a problem, SBB could be added after the incubation of the secondary antibody to optimize immunofluorescent labeling.

\section{Competing interests}

The authors declare that they have no competing interests.

Authors' contributions
\begin{tabular}{|l|c|c|}
\hline Authors' contributions & CJJ & JRS \\
\hline Research concept and design & $\checkmark$ & -- \\
\hline Collection and/or assembly of data & $\checkmark$ & -- \\
\hline Data analysis and interpretation & $\checkmark$ & -- \\
\hline Writing the article & $\checkmark$ & $\checkmark$ \\
\hline Critical revision of the article & $\checkmark$ & $\checkmark$ \\
\hline Final approval of article & $\checkmark$ & $\checkmark$ \\
\hline
\end{tabular}

\section{Acknowledgement}

The authors would like to thank Judith Stasko and Adrienne Shircliff of the National Animal Disease Center Histology and Microscopy Services Unit, for their technical experience and expertise.

\section{Publication history}

EIC: Gaetano Giuseppe Magro, University of Catania, Italy. Received: 01-Aug-2017 Final Revised: 12-Sep-2017

Accepted: 04-Oct-2017 Published: 15-Oct-2017

\section{References}

1. Schnell SA, Staines WA and Wessendorf MW. Reduction of lipofuscin-like autofluorescence in fluorescently labeled tissue. J Histochem Cytochem. 1999; 47:719-30. | Article | PubMed

2. Romijn HJ, van Uum JF, Breedijk I, Emmering J, Radu I and Pool CW. Double immunolabeling of neuropeptides in the human hypothalamus 
as analyzed by confocal laser scanning fluorescence microscopy. J Histochem Cytochem. 1999; 47:229-36. | Article | PubMed

3. Davis AS, Richter A, Becker S, Moyer JE, Sandouk A, Skinner J and Taubenberger JK. Characterizing and Diminishing Autofluorescence in Formalin-fixed Paraffin-embedded Human Respiratory Tissue. J Histochem Cytochem. 2014; 62:405-423. | Article | PubMed Abstract | PubMed FullText

4. Baschong W, Suetterlin R and Laeng RH. Control of autofluorescence of archival formaldehyde-fixed, paraffin-embedded tissue in confocal laser scanning microscopy (CLSM). J Histochem Cytochem. 2001; 49:1565-72. | Article | PubMed

5. Seeliger J and Moody DB. Monstrous Mycobacterial Lipids. Cell Chem Biol. 2016; 23:207-209. | Article | PubMed

6. Koronelli TV. Investigation of the lipids of saprophytic mycobacteria in the U.S.S.R. J Chromatogr. 1988; 440:479-86. | PubMed

7. Hartman TL. The use of Sudan Black B as a bacterial fat stain. Stain Technol. 1940; 15:23-8.

8. Burdon KL. Fatty Material in Bacteria and Fungi Revealed by Staining Dried, Fixed Slide Preparations. J Bacteriol. 1946; 52:665-78. | PubMed Abstract | PubMed FullText

9. Ortalo-Magne A, Lemassu A, Laneelle MA, Bardou F, Silve G, Gounon $P$, Marchal $G$ and Daffe M. Identification of the surface-exposed lipids on the cell envelopes of Mycobacterium tuberculosis and other mycobacterial species. J Bacteriol. 1996; 178:456-61. | Article | PubMed Abstract | PubMed FullText

10. Naser SA, Thanigachalam S, Spinelli N, Safavi MM, Naser N and Khan O. The 19kDa protein from Mycobacterium avium subspecies paratuberculosis is a glycolipoprotein. Adv Microbiol. 2013; 3:520-8. | $\underline{\text { Pdf }}$

11. Stabel JR, Ackermann MR and Goff JP. Comparison of polyclonal antibodies to three different preparations of Mycobacterium paratuberculosis in immunohistochemical diagnosis of Johne's disease in cattle. J Vet Diagn Invest. 1996; 8:469-73. | Article | PubMed

12. Elias JM. Immunohistopathology: A Practical Approach to Diagnosis. Chicago: American Society for Clinical Pathology Press, 2003.

\section{Citation:}

Jenvey CJ and Stabel JR. Sudan Black B masks Mycobacterium avium subspecies paratuberculosis immunofluorescent antibody labeling. J Histol Histopathol. 2017; 4:11.

http://dx.doi.org/10.7243/2055-091X-4-11 